\title{
DESIGNING EXPERIENCE OF AUTOMOTIVE TURBOCHARGER IMPELLER FOR FLANK MILLING
}

\author{
J.C. Bang ${ }^{* 1}$ and V.A. Shuripa ${ }^{2}$ \\ ${ }^{1}$ Dept. of Mechanical Engineering, Kumoh National Institute of Technology \\ ${ }^{2}$ Research and Development Dept., KEY YANG PRECISION CO., LTD
}

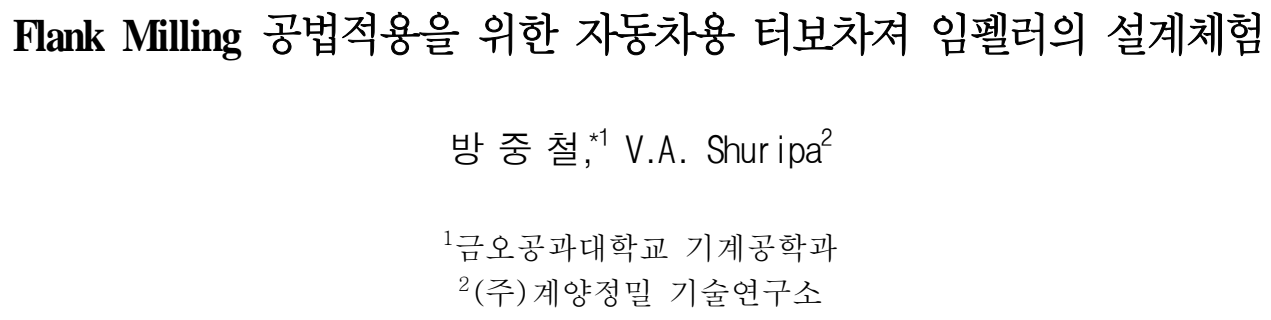

The performance of small-size impellers with ruled surfaces was investigated for flank milling over a wide speed range, using computational fluid dynamics analyses and gas bench tests. An impeller with a ruled surface was designed, manufactured, and tested to evaluate the effects of blade loading, the backsweep angle, and the relative velocity distribution on the compressor performance. The simulations and tests were completed using the same compressor cover with identical inlet and outlet channels to accurately compare the performance of the abovementioned impeller with a commercial impeller containing sculptured blades. Both impellers have the same number of blades, number of splitters, and shroud meridional profiles. The backsweep angles of the blades on the ruled impeller were selected to work with the same pinched diffuser as for a sculptured impeller. The inlet-to-exit relative velocity diffusion ratio and the blade loading were provided to maximize the flow rate and to minimize the surge flow rate. The design flow rate, rpm, were selected same for both impellers. Test results showed that for the compressor stage with a ruled impeller, the efficiency was increased by $0.32 \%$ with an extended surge margin without a reduction in the pressure ratio as compared to the impeller with the sculptured design. It was concluded that an increased relative velocity diffusion coupled with a large backsweep angle was an effective way to improve the compressor stage efficiency. Additionally, an appropriate blade loading distribution was important for achieving a wide operating range and higher efficiency.

Key Words : CFD(전산유체역학), Flank Milling(측면가공밀링), Turbocharger(과급기), Impeller(회전차), Compress Map(압축기 맵)

\section{Introduction}

Both sculptured and ruled surfaces for blades are widely used in centrifugal compressors. A sculptured surface is used for point milling, and a ruled surface is used for flank milling; these are the two common metal cutting methods for blades of impellers. In conventional Received: June 19, 2013, Revised: November 4, 2013, Accepted: November 4, 2013.

* Corresponding author, E-mail: bjc@kumoh.ac.kr DOI http://dx.doi.org/10.6112/kscfe.2013.18.4.001

(C) KSCFE 2013 flank milling, the entire blade surface is used after a single passage of the cutter through the blank material, engaging all cutting edges on the conical and ball-nose surfaces of the cutter. Regarding time expenditure and working cost, flank milling is an attractive method for the manufacturing of compressor impellers.

A blade with a sculptured surface does not have straight lines and cannot be created by a straight edge.

A ruled surface blade can be created by straight lines, called rulings, from points assigned on the hub side and the shroud side (Appendix 1). Ruled surfaces are frequently used for simplifying the blade surface design as 
compared to the free-form sculptured surfaces because of manufacturing costs[1].

The high-pressure, single-stage aluminum impellers used for turbochargers are typically sculptured because of their high-performance requirements. Sculptured impellers are increasingly used for compressors to meet energy saving requirements. Inherently, sculptured blade impellers provide superior characteristics as compared to ruled blade impellers. However, there is no definite reason why losses at the impeller channels cannot be reduced by a combination of the blade thickness distribution and the radial $(\beta)$ or angular $(\theta)$ distributions to approximate the performance of the sculptured impeller.

To reduce the differences in the performance between the blades with a sculptured surface and a ruled surface, an impeller with a ruled surface was fabricated with the same diameter, blade inlet width, blade exit width, and number of blades and splitters as a sculptured blade impeller. It was designed using a three-dimensional flow analysis method. The overall performance and the inlet and exit parts of each compressor were measured on gas bench; the performance differences among them were compared.

For comparative research, the sculptured blade commercial impeller was selected. The characteristics considered for comparison were averaged values for the backsweep angle, the inlet-to-exit relative velocity diffusion ratio, and the blade loading. The main objective was to demonstrate the difference between the flow parameters of the ruled impeller compressor and the sculptured impeller compressor; the ruled impeller compressor can be competitive with a commercial centrifugal compressor with a sculptured impeller in a high rotational speed range. By considering the effect of the modified blade geometry, the velocity triangles were revised to match the aerodynamic performance of the ruled impeller and diffuser.

\section{Design Tool}

Using the ANSYS Blade Generator software, the blades and the splitter were built using two layers, in contrast to the sculptured surfaces that were built using more than two layers. The layer is the curve line in the ANSYS Blade Generator software, which contains $\beta$ distributions, $\theta$ distributions, thickness values, and meridian positions. Based on the layer parameters and the distributions on the blade surfaces spanning between the hub and the shroud, the blade loading distributions and other fluid parameters were influenced by the direct correlation of the layer values.

Computational fluid dynamics (CFD) analyses were completed via a commercial code CFX-12.1 $1^{\mathrm{TM}}$, which solves the Reynolds-averaged Navier-Stokes equations numerically. The shear stress transfer turbulence model was employed for design point simulations, assuming a steady state in the present study. For defining the maximum flow rate, the $\mathrm{k}-\omega$ turbulence model was employed because of better convergence. The calculated regions included an inlet, one channel of an impeller, and the corresponding parts of a vane-less diffuser. The sector models were calculated for each component, and the models were periodically connected in the circumferential direction.

For comparison with a commercial impeller, the average fluid performances were evaluated at the inlet and exit of the impellers and at the exit of the diffuser. As the focus of the study, the blade loading distributions, span-wise from the hub to the shroud and stream-wise from the inlet to the exit, were represented through the fluid parameters of the impeller. The blade angle distribution on the hub and the shroud were specified from the loading distribution, the power, a work input coefficient, the volume flow rate, the triangles of average velocities, the pressure ratio, and other air parameters[2]. These parameters were considered to optimize the thickness and the $\beta$ and $\theta$ distributions for the blade and the splitters; these parameters were also used for the diffuser. To accept the design decisions in the operation range during modification of parameters on the hub and shroud curves in the ANSYS Blade Generator, the abovementioned performances were used for repeated simulations.

The number of the fine grid nodes was approximately 400000 , including $300000-350000$ grid points for the one channel of the impeller.

A grid refinement of 250000 nodes is recommended by CFX, as the number of nodes which is fine and is sufficient for engineering resolving of the flow features. Stator mesh had about 1800000 nodes, total assembly model had about 4200000 nodes (Appendix 1). Typical CFX templates of the boundary conditions were assigned based on the test results of the sculptured impeller compressor. The total pressure and total temperature were specified at the inlet boundary, and the mass flow rate was specified at the outlet boundary.

\section{Design Approach}

The comparison mode of the averaged values of the 


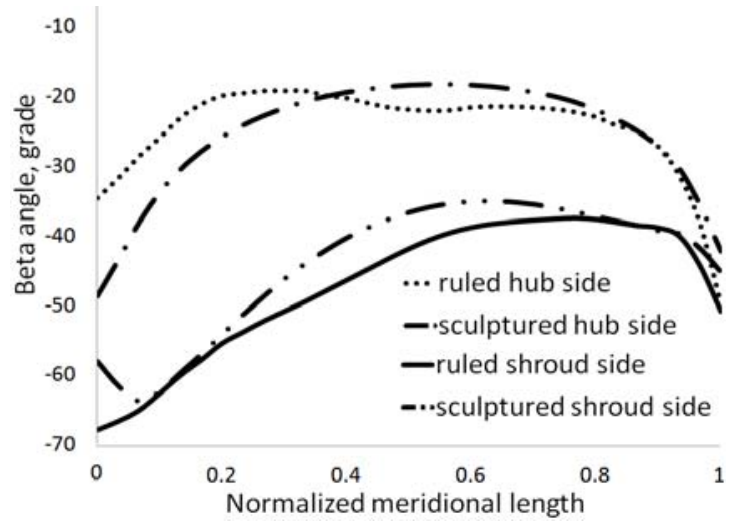

Fig. 1 Distribution of $\beta$ for ruled and sculptured blades of impellers (ANSYS Blade Generator)

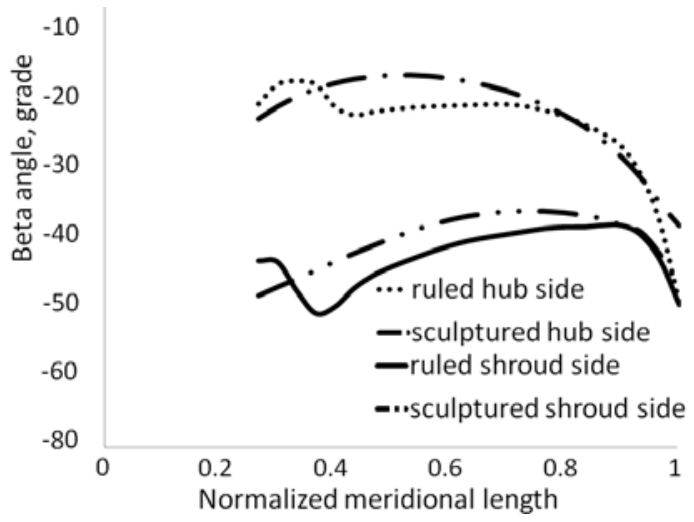

Fig. 2 Distribution of $\beta$ for ruled and sculptured splitters of impellers relative velocity and the pressure was used for the analysis with values obtained from the CFX result files[3]. For convenience, the comparison considered the blade and the splitter separately, even though the simulations were completed for the entire impeller, including both the blade and the splitter. The differences in the CFD parameters with the detailed countered presentation were not used because it involves too much CFD information. By comparison with the sculptured impeller, it is possible to make design decisions based on the integrated CFD parameters, which were obtained from the CFX result files. Because the $\beta$ distributions for the blade and the splitter for ruled surfaces are controlled by two layers and are assigned span-wise for the shroud and hub sides, the shapes are defined by combinations of geometrical parameters on the abovementioned two layers. The splitter angle distributions were selected to be independent from the blade angle distributions.

For commercial impellers, the blade and splitter $\beta$ distributions for blade surfaces are controlled by five layers and are assigned on the shroud and hub sides with three intermediate layers of general (sculptured) span-wise layers. These surface shapes are defined by geometrical parameters of the five layers. Splitter angle distributions were selected to be independent from angle distributions of the main blade and are also controlled by five layers.

The ruled blade has larger absolute values of the $\beta$ angle on the shroud side (Fig. 1) than the inlet of the sculptured blade, but it has a smaller value on the hub side to increase the blade loading on the tip of the inducer.

The distribution of $\beta$ for the shroud and the hub for the ruled blade has a strong influence on the blade in the span-wise direction; it is required that the blade-to-blade load provides a flow with secondary streams, which do not harm efficiency. The blade's $\beta$ distribution on shroud side of the ruled impeller smaller than the $\beta$ for the sculptured impeller between normalized lengths of 0.15 and 0.8 ; from 0 to 0.15 and from 0.8 to 1 , there is no difference in the $\beta$ distribution. The $\beta$ angle for the ruled blades close to the impeller exit was higher than $\beta$ for the sculptured blades, which increased backswept angle in considered case improves interaction with diffuser.

At the inlet of the channel, the ruled splitter has a smaller absolute values $\beta$ on the shroud side and the hub side (Fig. 2) than the sculptured splitter to increase the blade loading on the tip, which is the pressured side of the blade. The splitter $\beta$ distribution on the shroud side of the ruled impeller is lower than the sculptured impeller within the normalized meridional length range $0.3-0.8$ and is the same in the range 0.8-0.85. In result on exit part of impellers the absolute values of $\beta$-angles for ruled surfaces are larger than for sculptured surfaces. These characteristics are mainly for fitting with the blade, for more even turning of the flow, and for more even radial outflow, because increasing angles near the exit insignificantly increases the backsweep of the splitter.

The distributions of the thicknesses are similiar for both types of blades (Fig. 3). The thickness of the ruled blade is selected to be smaller than that of the sculptured blade. The distribution shows that the maximum value of the normalized length is at approximately 0.25 from the inlet of the impeller channel for both impellers. Near the exit, the thickness of the ruled surface is slightly higher than the thickness for the sculptured surface. The thicknesses on span 0.5 for both impellers are not represented, but for the ruled blade, the values are smaller and the distribution follows for the hub side distribution tendency. 


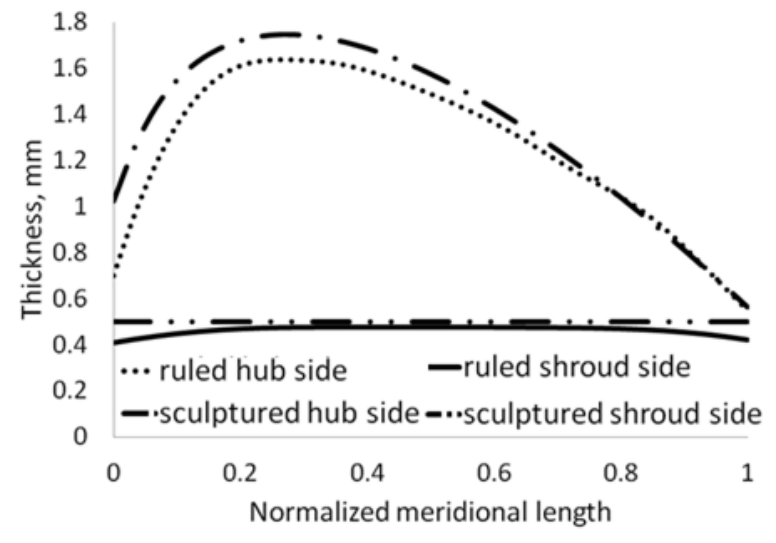

Fig. 3 Distribution of thicknesses for ruled and sculptured blades of impellers

The distributions of the thicknesses are similiar for the shroud side for both types of splitters (Fig. 4). The thickness of the ruled splitter on hub side is selected to be smaller than that for the sculptured splitter; the maximum thickness is approximately at 0.75 . The positions and values of the maximum were selected to increase the efficiency and to moderate the diffusion before exiting the impeller.

\section{Results and Discussion}

The results of the simulations were considered from viewpoint of the summarized performances and in comparative mode owing to designing simulations for one sector of impellers having a configuration of six blades and six splitters. A sector, as mentioned above, includes an impeller and a diffuser. The main design was based on the results of the simulations at a design point, near the area of maximum efficiency of a commercial compressor map at $157,500 \mathrm{rpm}$, with a volume flow rate of 0.0597 $\mathrm{m}^{3} / \mathrm{s}$ and an inlet air temperature of $20^{\circ} \mathrm{C}$. That particular simulation point is different from the point on the compressor map, but was selected because it only includes one channel and a minimal amount of calculation time.

A selected combination of $\beta$ and thickness distributions should provide the necessary load for the blades. As mentioned above, at the exit of the impellers, $\beta$ for ruled surfaces was larger than for sculptured blades for avoiding a large turning of the flow in the channel and for obtaining a higher radial outflow at the impeller exit. Thus, less work is performed on tangential acceleration. If the blade angle turns radically at the inlet, the length of the passage increases. If the blade is turned abruptly at the exit to meet the desired backsweep, the positive

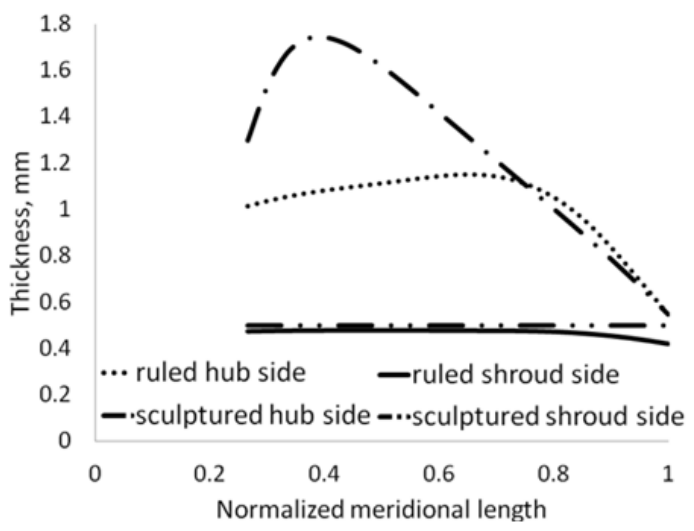

Fig. 4 Distribution of thicknesses for ruled and sculptured splitters of impellers

effects of the backsweep will be lost because the flow will separate from the blade surface. For conditions near the area of maximum efficiency of the compressor, the angle of the average relative velocity at the exit of the ruled blade was $\beta_{2}=-48^{\circ}$, which is larger than the angle for the sculptured impeller angle, $\beta_{2}=-45^{\circ}$. That selection was made to provide stability of the compressor near the surge line at all rotation speeds without reducing the maximum flow rate. In design-off conditions flow, the backsweep difference is smaller than $1.0^{\circ}$.

The $\beta$ distribution of the blade in combination with the thickness distribution provided the configuration of the inducer in the impeller channel, which avoids large amounts of diffusion at a small volume flow rate, which can create adverse operation conditions in stall and surge.

The $\beta$ distribution for the ruled blade is controlled on two layers only and the curvature of the suction surface of the inducer cannot be directly provided on span near the shroud side. In that case, it is reasonable to combine the blade's $\beta$ and thickness distributions on the shroud and hub sides to provide a suction surface curvature with the ability to make a suitable throat area. To make this combination available, the thicknesses of the blade on the hub and shroud sides were reduced while reducing $\beta$ on the hub side. The stress and modal performances were not deteriorated. The surge limit of the ruled blade should be considered carefully during the construction of the blade surface. The geometrical characteristics that define the surge limit, such as the throat area position, were selected to maximize the flow rate. For sculptured blades, the throat area was $497 \mathrm{~mm}^{2}$, and for ruled surfaces, the throat was $489 \mathrm{~mm}^{2}$, which is $1.6 \%$ smaller.

In the results, the specified $\beta$ distributions provide the conditions near the area of maximum efficiency; here, the 


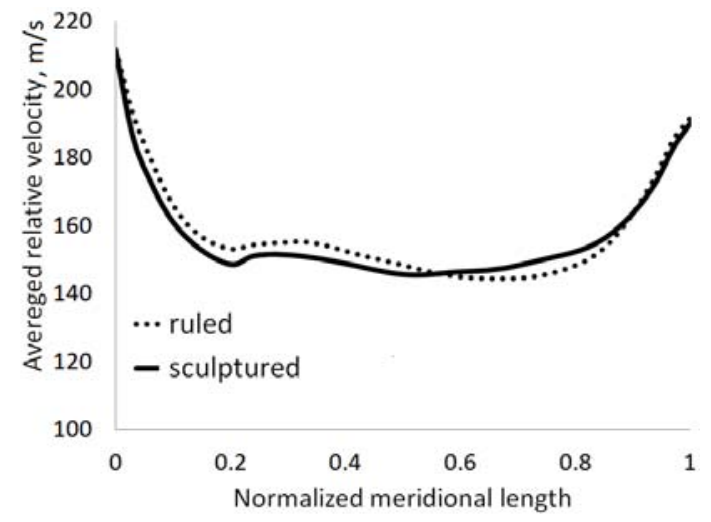

Fig. 5 Distribution of averaged relative velocity for ruled and sculptured blades of impellers
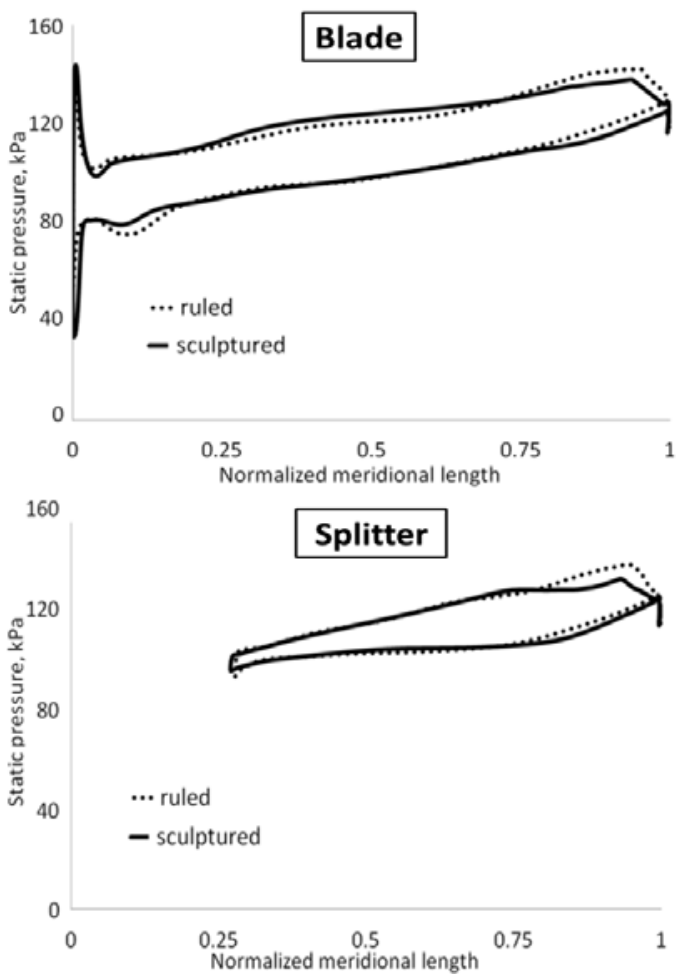

Fig. 6 Distribution near shroud of static pressure for ruled and sculptured blades and splitters of impellers

relative velocity is averaged by the channel area, which is shown in Fig. 5.

At the inlet of the impeller channel, the relative velocity is lower for sculptured blades than for ruled blades. For sculptured blades, the $\beta$ distributions provide a higher level of diffusion at the inlet of the impeller. At the outlet of the impeller, the average relative velocity in the channels with ruled blades is lower and has a higher level of diffusion. The consequence of the diffusion
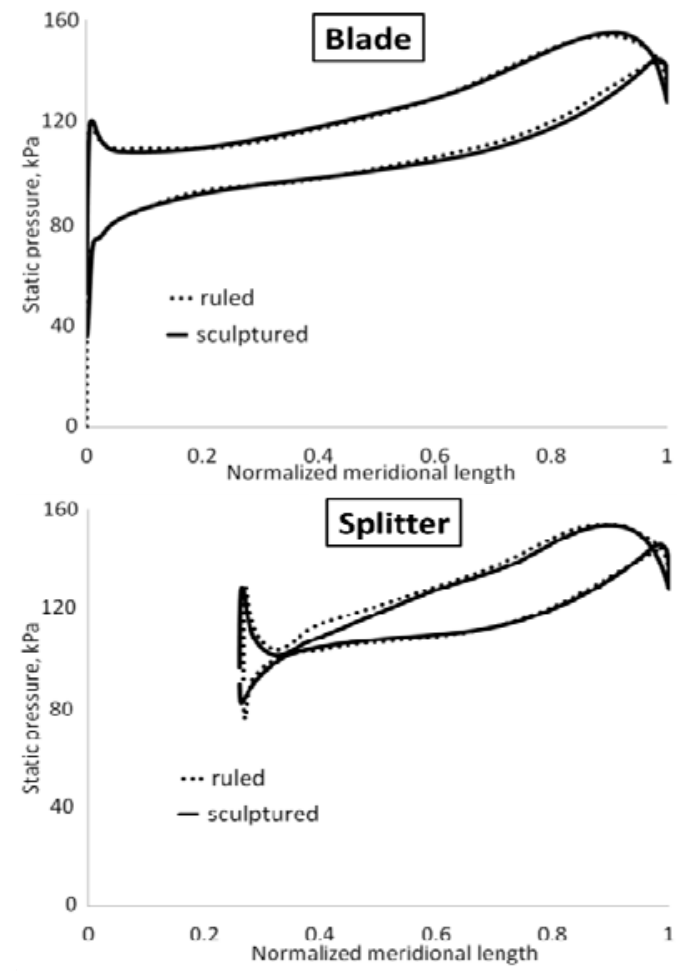

Fig. 7 Distribution of middle span of static pressure for ruled and sculptured blades and splitters of impellers

contributes to an increasing share of centrifugal forces to create static pressure that limits the surge in the diffuser of the compressor[4].

Fig. 6 shows the static pressure in the inlet of the channel with respect to the normalized length. On the low-pressure portion at approximately 0.1 , the pressure is lower for the ruled blade on suctioned surfaces than for the sculptured blade. On the high pressure side at approximately 0.1 , the static pressure is higher for the ruled blade than for the sculptured blade. At normalized lengths from 0.25 to 0.75 , the static pressure is lower for ruled blade than for the sculptured blade. At normalized lengths above 0.8 (the exit of the channel) on the high pressure side, the static pressure is larger for the ruled blade than for the sculptured blade.

For the splitter, the static pressure at the exit near the shroud (normalized lengths between 0.8 and 1.0) on the high pressure surface is larger for the ruled blade than for the sculptured blade (Fig. 6). Near the shroud for the low-pressure surface, the diffusion is higher for the ruled blade than for the sculptured blade at the inlet part and the exit of the channel.

Fig. 7 shows that the static pressure at the inlet of the channel at normalized lengths of approximately 0.5 to the 

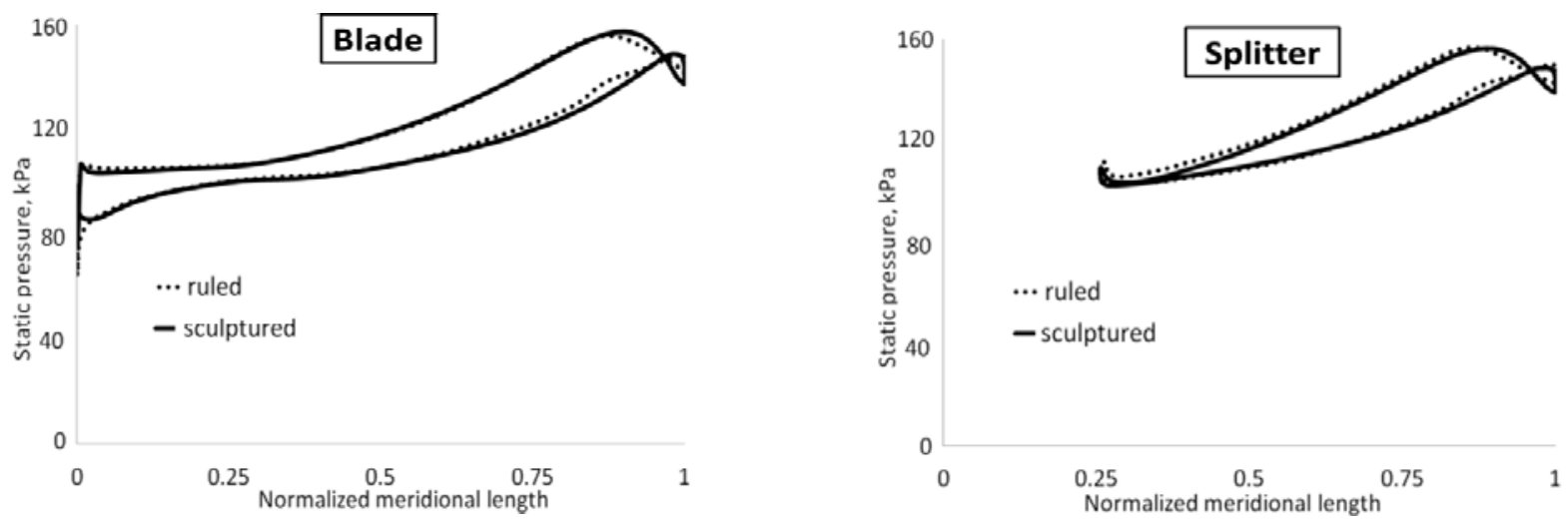

Fig. 8 Distribution of static pressure on hub side for ruled and sculptured blades and splitters of impellers

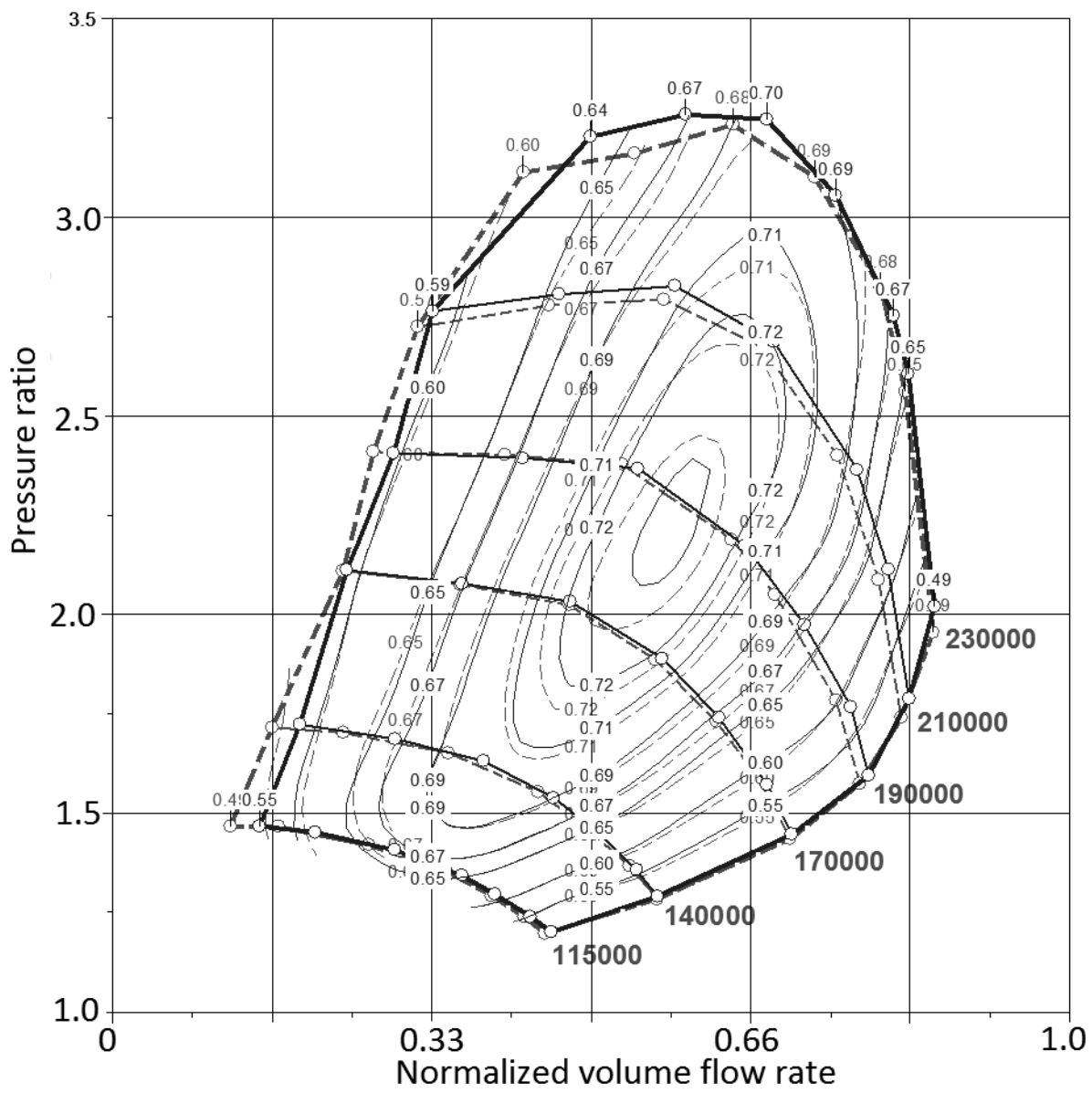

Fig. 9 Maps of ruled and sculptured compressors in comparative overlapping. The dash lines represent the impeller with the ruled blades (The impeller diameter is $44 \mathrm{~mm}$ )

exit is larger for the ruled blade on a suctioned surface than for the sculptured blade. On the high-pressure surface, the static pressure is almost same for both types. For the splitter on the high-pressure side, the static pressure is larger for the ruled blade than for the sculptured blade along the entire normalized length of the splitter (Fig. 7). This means that the channel located between the suction side of the blade and the pressure side of the splitter in the middle has a higher level of diffusion for the ruled blade than for the sculptured blade of the impeller.

Fig. 8 shows that the hub side static pressure at the 
inlet of the channel at normalized lengths of approximately 0.5 to the exit is larger for the ruled blade on the suctioned surfaces than for the sculptured blade. On the high-pressure side, the static pressure is approximately same for both types. At the middle of the high-pressure side, the static pressure is higher for the ruled blade than for the sculptured blade along the entire normalized length of the splitter and at the suctioned surface near the exit (Fig. 8). This means that the channel, which is located between the suctioned side of the blade and the pressure side of the splitter on the hub side, has a higher level of diffusion for the ruled blade than for the sculptured blade of the impeller.

In Fig. 9, the efficiency lines show that high efficiency values encompass a larger area for the ruled impeller than for the sculptured impeller. The ruled impeller has higher maximum efficiency than the sculptured impeller for $0.32 \%$. The location of the surge line is at a lower volume flow rate for the ruled impeller. The maximum volume flow rate is the same for both compressors, even though the ruled impeller has a throat area that is $1.6 \%$ smaller. The same maximum flow rate with a reduced throat area was achieved by reducing the thickness of the ruled splitter. The pressure ratio values on the speed line for both compressors have approximately the same flow rate from the surge line to the maximum efficiency. Only at $210000 \mathrm{rpm}$ is the pressure ratio lower for the ruled impeller for all flow rates.

For the application of an automotive turbocharger, the higher efficiency values in the range between the surge line and the field of maximum efficiency provide better engine performances regarding low-end torque and partial-load operation conditions. In that case of sculptured blades the impeller has strict limitations. Sculptured impeller can be manufactured by casting or point milling methods. For obtaining high speed operation condition (impeller tip speed $560 \mathrm{~m} / \mathrm{s}$ ) aluminum casting impeller has insufficient strength. For high speed are applied impellers by the point milling method too time is necessary. For example, to make sculptured impeller it is necessary to spend about 40 minutes, to make ruled impeller-about 4 minutes.

For these purposes, the designed ruled impeller outperforms the compared sculptured impeller.

\section{Conclusions}

(1) The $\beta$ distributions for the blade and the splitter for ruled surfaces are controlled by two layers and are assigned on the shroud and hub sides with a ruled span-wise type. The ruled blades had larger absolute values of $\beta$ on the shroud side than the sculptured blades at the inlet of the channel to increase the blade loading on the tip of the inducer.

(2) The $\beta$ and thickness distributions defined at the inlet of the ruled impeller channel reduced the relative velocity less than for the sculptured blade surfaces. At the outlet of the impeller, the average relative velocity in the channels with ruled surfaces blades was lower with a higher level of diffusion. On the high-pressure side, the static pressure is larger for the ruled blades than for the sculptured blades at a normalized length close to 0.1 .

(3) For the ruled impeller, the efficiency was $0.32 \%$ higher (with an increasing surge margin) than for the sculptured impeller with the same maximum flow rate due improved relative velocity distribution.

(4) The $\beta$ and thickness distributions enable the ruled impeller to be competitive with the performances of sculptured impellers.

\section{Acknowledgements}

This paper was supported by Research Fund, Kumoh National Institute of Technology

\section{References}

[1] 2002, Tsay, D.M., Chen, H.C. and Her, M.J., "A study of five flank machining of centrifugal compressor impellers," Journal of Engineering for Gas Turbines and Power, Vol.124, pp.177-181.

[2] 2004, Zangeneh, M., Schleer, M., Ploger, F., Hong, S.S., Ribi, B. and Abhari, R.S., "Investigation of an inversely designed centrifugal compressor stage-Part 1 : Design and numerical verification," Journal of Turbomachinery, Vol.126, pp.73-81.

[3] 2011, Shibata, T., Yagi, M., Nishida, H., Kobayashi, H. and Tanaka, M., "Performance improvement of a centrifugal compressor stage by increasing degree of reaction and optimizing blade loading of a 3D impeller," Journal of Turbomachinery, Vol.133, pp.021004 (1-8).

[4] 2010, Zheng, X.Q., Zhang, Y.J. and Yang, M.Y., "Research and development on transonic compressor of high pressure ratio turbocharger for vehicle internal combustion engines," Science China Technological Sciences, Vol.13, No.4, pp.1817-1823. 


\section{Appendix 1}

In Fig. A1 the sculptured (left) and the ruled (right) blades impellers are represented. The sculptured blades and splitters have curved leading edges but ruled blades have straight leading edges.

In Fig. A2 the mesh of compressor assembly (about 4200000) is represented. To design ruled impeller the blades and the splitters were modified to find out shape, which provides performances better than sculptured impeller. In these cases number of mesh nodes was slightly changed.
Appendix 2

A similar approach was applied for designing the ruled impeller with a $56 \mathrm{~mm}$ diameter, which is going to be used instead of a commercial sculptured impeller having the same diameter. The maps are shown in Fig. A3. Both impellers, with ruled and with sculptured blades, were tested with the same compressor cover under the same conditions. In Fig. A3, the compressor with the ruled impeller has maximum efficiency more than $1 \%$ higher than the compressor with the sculptured impeller. Beside that mainly in engine operation range the lines of constant efficiency having higher values overtake the larger area for designed ruled impellers in comparison with the sculptured impeller. The operation flow rates are same for both compressors.

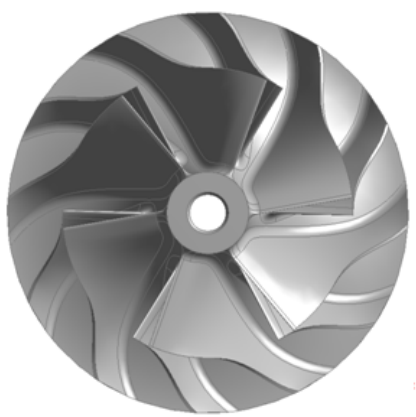

Fig. A1 The sculptured (left) and the ruled (right) blades impellers

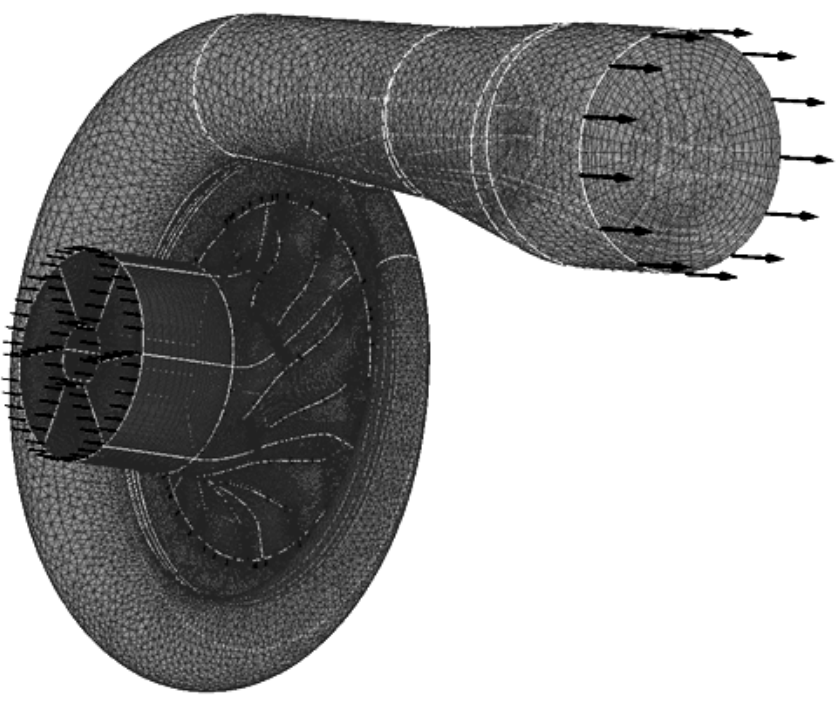

Fig. A2 Mesh of compressor assembly (about 420000)

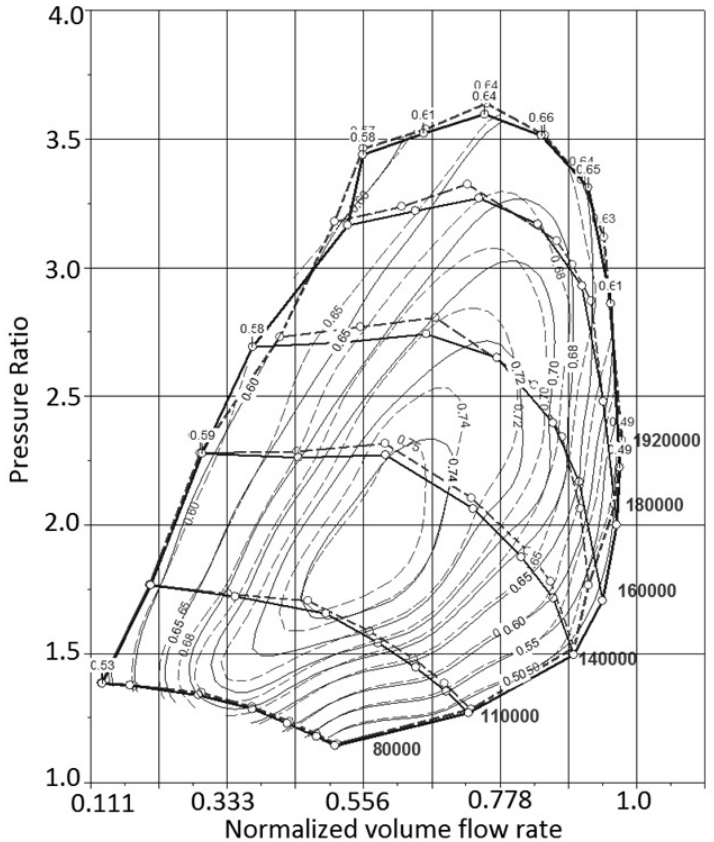

Fig. A3 Maps of ruled and sculptured compressors in comparative overlapping, dash lines represent compressor with ruled blades 